\title{
Revisiting the two-side optimization problem in satellite pursuit-evasion
}

\author{
KeKe Zhang ${ }^{1,2,3}$, ZhongCheng $\mathrm{Mu}^{3,}$ a , Dong $\mathrm{Ye}^{4}$, JinLing $\mathrm{Qi}^{3}$ \\ ${ }^{1}$ ShangHai Institute of Microsystem and Information Technology, ShangHai 200050, China \\ ${ }^{2}$ University of Chinese Academy of Sciences, BeiJing 100049, China \\ ${ }^{3}$ ShangHai Engineering Center for Microsatellite, ShangHai 201210, China \\ ${ }^{4}$ Harbin Institute of Technology, Harbin 150001, China
}

\begin{abstract}
In the present paper two-side optimization problem where the intercepting satellite pursuits the target satellite was studied. In the pursuit-evasion game the continuous thruster and the simple central gravity with J2 perturbation were involved for two satellites. With applying the functional extremum condition, the optimal control outputs of both side satellites were obtained by building the systems Hamilton function. Further, the two side optimal problem was transformed to the TPBVP, which contributed the solution with the mixed numerical method. Finally, the proposed method was validated by the numerical simulation, where the optimal interception and escaping trajectories were illustrated.
\end{abstract}

\section{Introduction}

In previous studies, for the satellite interception question, the targets were assumed generally to be static without any motion ability and moved in the pre-assigned orbits. Thus the solver only needs to design a maneuvering trajectory for the attacking satellite in solving the interception which could be regarded as single side optimization question. The design and optimization method was different as the propulsive force exerting on the interceptor. Luo, etc. studied the multiple impulsive fuel optimal orbit rendezvous problem under the linear [1] and nonlinear [2] dynamics, they directly applied the simplex, quadratic, genetic [3] and simulated annealing algorithms [4] to optimize the impulse vectors. Simulation results show that combining with the meta optimization method and the SQP method can effectively solve the problem [5]. Lawden, etc. proposed the principal vector theory to determine the trajectory optimal property [6] and added the coast stage or middle impulse to achieve the fuel optimal trajectory [7]. For the continuous thruster, the indirect method was applied based on the calculus of variation and maximum principle, causing that the interception question was transformed to the two point boundary value problem (TPBVP). Greenwood solved the transformed problem by the shooting method and applied it to study the small thrust fuel optimal transferring problem in the co-plane orbit [8]. Unfortunately, the initial value of the indirect method is difficult to estimate and is sensitive for the result achievement. The direct method scatters the total transferring procedure to small steps and transfers the dynamical optimization to the constrained minimum solving problem. Hargraves, etc. put forward the collocation method to solve the time optimal interception climbs problem [9]. Enright [10]

\footnotetext{
${ }^{\text {a }}$ Corresponding author : muzhongcheng@aliyun.com
} 
improved the method and reduced the computing scale by developing the nonlinear programming with the parallel shooting frame. Herman, etc. [11] proposed the high order collocation method which was widely used to solve various earth transferring optimal orbits.

Contrarily, for the scenarios with having the orbit maneuvering ability, the method described above may become infeasible since both the interceptor and the target are in the non-stationary state. Considering both sides optimal features, the designer needs to give the control method, where the target satellite could escape from the interceptor when it discovered the later attacking intention. Actually, it is a classical pursuit-evasion problem and can be coped with the game theory. Iaacs firstly concentrated on this problem and defined the two side optimal solution as the saddle solution [12]. For the realistic situation, the saddle solution is difficult to get the solution since the system dynamics cover two players and the dynamics often are highly nonlinear and complex. Anderson [13] solved the coplanar spacecraft pursuit-evasion with large thrust by finding the difference between the current state and the pre-calculating state to update the conjugate. Menon [14] obtained the feedback control strategy for the spacecraft with saturated output by applying the back-step method and the quadratic objective function. Mauro Pontani $[15,16]$ transferred the original interception problem to the TPBVP and proposed the semi-direct method to solve it. This method was used in intercepting an extraatmosphere missile and two spacecrafts attack-escape. Stupik [17] studied satellite combat based on the CW equation with the help of Kriging method. Great many standard solutions with various initial conditions solved with the former method were interpolated to obtain the suboptimal feedback solution when initial states domain and the disturbance were finite.

On the other hand, earth non-spherical perturbation has a great influence on the low orbital satellite motion. No matter how accurate the initial trajectory was designed, the satellite can still drifted an intolerable distance. Thus the present paper mainly studied the pursuit-evasion game of two satellites with J2 perturbation effect, where both satellites carried the continuous thruster with limited magnitude to perform orbital maneuver.

\section{Pursuit and Evasion Dynamics with J2 Perturbation}

This intercepting event ends when the pursuer catches the target, namely both satellites arrives at the same location in the space. During the catching and escaping process, the pursuer adopts the control strategy to catch the target as soon as possible while the later tries its best to postpone the intercept terminal time. In order to make the question simple, it was assumed that both players can completely acquire the counter dynamics information.

Firstly, aiming to complete the interception, it is required that UP (the specific thrusts of the interceptor) is bigger than UE (the specific thrusts of the escaper). Mauro [16] used a group of new state variables to describe the spacecraft's motion to make the simulation more real. Thus the same variables were adopted in the paper. The state variables of the pursuer and evader can be defined as $\left(r_{i}, \xi_{i}, \varphi_{i}, \gamma_{i}, v_{i}, \zeta_{i}\right)$ with representing respectively the satellite's geocentric distance, geometrical longitude, latitude, path angle, speed and azimuth angle, and $i=P, E$ stand for the two sides in the interception, respectively.

The simple two-body gravity exerts a centripetal force on the satellite which produces an acceleration vectors can been represent in the geodetic coordinate system as following

$$
\boldsymbol{a}_{\mu}=\left[\begin{array}{ccc}
-\frac{\mu}{r^{2}} & 0 & 0
\end{array}\right]^{T}
$$

where $\mu$ represents the gravitational constant of the earth.

The acceleration arose by the $\mathrm{J} 2$ non-spherical perturbation can been decomposed in the same coordinate as below 


$$
\boldsymbol{a}_{J_{2}}=-\frac{3 J_{2} \mu R_{e}^{2}}{2 r_{4}}\left[\begin{array}{c}
1-3 \sin ^{2} \varphi \\
2 \sin \varphi \cos \varphi \\
0
\end{array}\right]
$$

where $J_{2}=1.018 \times 10^{-3}$ is the $\mathrm{J} 2$ perturbation constant and $R_{e}=6378.165 \mathrm{~km}$ is the mean earth radius. Using the coordinates transformation relation, the dynamics of the satellite can be given as equation (3), where $G_{J 2}=J_{2} \mu R_{e}^{2}$.

$$
\begin{aligned}
& \dot{r}=v \sin \gamma \\
& \dot{\xi}=\frac{v \cos \gamma \cos \zeta}{r \cos \varphi} \\
& \dot{\varphi}=\frac{v \cos \gamma \sin \zeta}{r} \\
& \dot{\gamma}=\frac{v}{r} \cos \gamma+\frac{U \cos \beta \sin \alpha}{v}-\frac{\mu \sin \gamma}{r^{2} v}-\frac{3 G_{J 2}}{4 r^{4} v}\{\sin \gamma[3 \cos (2 \varphi)-1]+2 \cos \gamma \sin \zeta \sin (2 \varphi)\} \\
& \dot{v}=U \cos \beta \cos \alpha-\frac{\mu \sin \gamma}{r^{2}}-\frac{3 G_{J 2}}{4 r^{4}}\{\cos \gamma[3 \cos (2 \varphi)-1]+2 \sin \gamma \sin \zeta \sin (2 \varphi)\} \\
& \dot{\zeta}=\frac{U \sin \beta}{v \cos \gamma}-\frac{v \tan \varphi \cos \gamma \cos \zeta}{r}-\frac{3 G_{J 2}}{2 r^{4} v \cos \gamma} \cos \zeta \sin (2 \varphi)
\end{aligned}
$$

Let the states of the interceptor $(\mathrm{P})$ and target $(\mathrm{E})$ be

$$
\begin{aligned}
\boldsymbol{x}_{P} & =\left[\begin{array}{llllll}
x_{1} & x_{2} & x_{3} & x_{4} & x_{5} & x_{6}
\end{array}\right]^{T} \\
& =\left[\begin{array}{llllll}
r_{P} & \xi_{P} & \varphi_{P} & \gamma_{P} & v_{P} & \zeta_{P}
\end{array}\right]^{T} \\
\boldsymbol{x}_{E} & =\left[\begin{array}{llllll}
x_{7} & x_{8} & x_{9} & x_{10} & x_{11} & x_{12}
\end{array}\right]^{T} \\
& =\left[\begin{array}{llllll}
r_{E} & \xi_{E} & \varphi_{E} & \gamma_{E} & v_{E} & \zeta_{E}
\end{array}\right]^{T}
\end{aligned}
$$

Obviously the pursuer and evader optimize the angle of the thruster to compete for the interception ending time, thus the control variables of satellites are given as below

$$
\begin{aligned}
& \boldsymbol{u}_{P}=\left[\begin{array}{ll}
u_{1} & u_{2}
\end{array}\right]^{T}=\left[\begin{array}{ll}
\alpha_{P} & \beta_{P}
\end{array}\right]^{T} \\
& \boldsymbol{u}_{E}=\left[\begin{array}{ll}
u_{3} & u_{4}
\end{array}\right]^{T}=\left[\begin{array}{ll}
\alpha_{E} & \beta_{E}
\end{array}\right]^{T}
\end{aligned}
$$

Further, the dynamics of both sides can be rewritten as below

$$
\begin{aligned}
& \dot{\boldsymbol{x}}_{P}=\boldsymbol{f}_{P}\left(\boldsymbol{x}_{P}, \boldsymbol{u}_{P}, t\right) \\
& \dot{\boldsymbol{x}}_{E}=\boldsymbol{f}_{E}\left(\boldsymbol{x}_{E}, \boldsymbol{u}_{E}, t\right)
\end{aligned}
$$

Interception ending requires that the location coordinate of both satellites is equal, namely the following conditions are satisfied at the terminal time $t_{f}$

$$
\begin{aligned}
& r_{P}=\left.r_{E}\right|_{t=t_{f}} \Rightarrow x_{1}\left(t_{f}\right)-x_{7}\left(t_{f}\right)=0 \\
& \xi_{P}=\left.\xi_{E}\right|_{t=t_{f}} \Rightarrow x_{2}\left(t_{f}\right)-x_{8}\left(t_{f}\right)=0 \\
& \varphi_{P}=\left.\varphi_{E}\right|_{t=t_{f}} \Rightarrow x_{3}\left(t_{f}\right)-x_{9}\left(t_{f}\right)=0
\end{aligned}
$$


Rewritten them to a compact vector form as following

$$
\left.\boldsymbol{N}(\boldsymbol{x}, t)\right|_{t=t_{f}}=0
$$

Finally, the orbital elements can be transformed to the state variables according to the relation equation in reference [13].

\section{Saddel Solution of the Pursuit-evasion Game}

In terms of the pursuer, the interception ending time is expected to become short as little as possible, while the counterpart escapes the catch to the best of its ability. Thus the ending time can be chosen as the objective namely

$$
J=t_{f}
$$

This interception is a traditional zero sum differential game. When the control strategies variables pair $\left(\boldsymbol{U}_{P}, \boldsymbol{U}_{E}\right)$ satisfies the condition below, it is defined as the saddle solution $\left(\boldsymbol{U}_{P}^{*}, \boldsymbol{U}_{E}^{*}\right)$

$$
J\left(\boldsymbol{U}_{P}^{*}, \boldsymbol{U}_{E}\right) \leq J\left(\boldsymbol{U}_{P}^{*}, \boldsymbol{U}_{E}^{*}\right) \leq J\left(\boldsymbol{U}_{P}, \boldsymbol{U}_{E}^{*}\right) \quad \forall \boldsymbol{U}_{P}, \boldsymbol{U}_{E}
$$

which means that if only the purser adopts the optimal control strategy, the objective will be better than that they both make use the optimal control strategies, and the interception ending time will shorten. Otherwise, the time will increase.

The open loop solution of the differential game is obtained based on the initial states $\boldsymbol{x}_{0}$ of both sides and the current time, whilst the close loop solution which is more flexible to the real situation can be solved according to the initial states, current time and the current states. Conclusion has been made that if the value function is continuous in the admissible domain of control strategies, the open loop solution and close loop solution are identical [18]. And for the system dynamics with high complexity and nonlinearity, it is almost impossible to analytically solve the saddle solution. Thus obtaining the close loop solution is unpractical currently, the optional way is to solve the open loop control strategy and optimal trajectory relevant.

For the two-side dynamical optimization problem with system (5), Maya type objective (8) and terminal condition collection (7), the Hamiltonian function can be defined as the optimal control theory

$$
H=\lambda_{P}^{T} \boldsymbol{f}_{P}+\lambda_{E}^{T} \boldsymbol{f}_{E}=H_{P}+H_{E}
$$

where $\lambda_{P}$ and $\lambda_{E}$ stand for the conjugates of the pursuer and evader's state variables, respectively. $H_{P}$ is given as equation (12)

$$
\begin{aligned}
H_{P}= & \lambda_{1} v_{P} \sin \gamma_{P}+\lambda_{2} \frac{v_{i} \cos \gamma_{P} \cos \xi_{i}}{r_{P}}+\lambda_{3} \frac{v_{P} \cos \gamma_{P} \sin \xi_{P}}{r_{P} \cos \varphi_{P}}+\lambda_{4}\left(\frac{v_{P} \cos \gamma_{P}}{r_{P}}-\frac{\mu \sin \gamma_{P}}{r_{P}^{2} v_{P}}\right. \\
& -\frac{3 G_{J 2}}{4 r_{P}^{4} v_{P}}\left\{\sin \gamma_{P}\left[3 \cos \left(2 \varphi_{P}\right)-1\right]+2 \cos \gamma_{P} \sin \zeta_{P} \sin \left(2 \varphi_{P}\right)\right\}+\frac{U_{P} \cos \beta_{P} \sin \alpha_{P}}{v_{P}} \\
& +\lambda_{5}\left(U_{P} \cos \beta_{P} \cos \alpha_{P}-\frac{3 G_{J 2}}{4 r_{P}^{4}}\left\{\cos \gamma_{P}\left[3 \cos \left(2 \varphi_{P}\right)-1\right]+2 \sin \gamma_{P} \sin \zeta_{P} \sin \left(2 \varphi_{P}\right)\right\}\right. \\
& \left.-\frac{\mu \sin \gamma_{P}}{r_{P}^{2}}\right)+\lambda_{6}\left(\frac{U_{P} \sin \beta_{P}}{v_{P} \cos \gamma_{P}}-\frac{3 G_{J 2} \cos \zeta_{P} \sin \left(2 \varphi_{P}\right)}{2 r_{P}^{4} v_{P} \cos \gamma_{P}}-\frac{v_{P} \tan \varphi_{P} \cos \gamma_{P} \cos \zeta_{P}}{r_{P}}\right)
\end{aligned}
$$


$H_{E}$ is like $H_{P}$ with the subscript substituting to be $E$ and the conjugates changing from $\lambda_{i}, i=1,2, \ldots, 6$ to $\lambda_{i+6}$.

If defining the co-state variables of terminal conditions (7) as $v=\left[\begin{array}{lll}v_{1} & v_{2} & v_{3}\end{array}\right]^{T}$, the conjugates at terminal time can be obtained with satisfying the conditions as equations (13)

$$
\begin{aligned}
& \lambda_{P}^{T}=\frac{\partial \varphi\left(t_{f}, \boldsymbol{x}\left(t_{f}\right)\right)}{\partial \boldsymbol{x}_{P}\left(t_{f}\right)}+\left.\frac{\partial \boldsymbol{N}^{T}}{\boldsymbol{x}_{P}}\right|_{t=t_{f}} \\
& \lambda_{E}^{T}=\frac{\partial \varphi\left(t_{f}, \boldsymbol{x}\left(t_{f}\right)\right)}{\partial \boldsymbol{x}_{E}\left(t_{f}\right)}+\left.\frac{\partial \boldsymbol{N}^{T}}{\boldsymbol{x}_{E}}\right|_{t=t_{f}}
\end{aligned}
$$

Substituting the correlative variables and equations, $\lambda\left(t_{f}\right)$ is given below

$$
\begin{aligned}
& \left\{\begin{array}{l}
\lambda_{1}\left(t_{f}\right)=v_{1} \\
\lambda_{2}\left(t_{f}\right)=v_{2} \\
\lambda_{3}\left(t_{f}\right)=v_{3} \\
\lambda_{4}\left(t_{f}\right)=\lambda_{5}\left(t_{f}\right)=\lambda_{6}\left(t_{f}\right)=0
\end{array}\right. \\
& \left\{\begin{array}{l}
\lambda_{7}\left(t_{f}\right)=-v_{1} \\
\lambda_{8}\left(t_{f}\right)=-v_{2} \\
\lambda_{9}\left(t_{f}\right)=-v_{3} \\
\lambda_{10}\left(t_{f}\right)=\lambda_{11}\left(t_{f}\right)=\lambda_{12}\left(t_{f}\right)=0
\end{array}\right.
\end{aligned}
$$

And collecting the equations, and the conjugates satisfy the following conditions

$$
\begin{aligned}
\lambda_{4}\left(t_{f}\right)= & \lambda_{5}\left(t_{f}\right)=\lambda_{6}\left(t_{f}\right)=\lambda_{10}\left(t_{f}\right) \\
= & \lambda_{11}\left(t_{f}\right)=\lambda_{12}\left(t_{f}\right)=0 \\
& \lambda_{1}\left(t_{f}\right)+\lambda_{7}\left(t_{f}\right)=0 \\
& \lambda_{2}\left(t_{f}\right)+\lambda_{8}\left(t_{f}\right)=0 \\
& \lambda_{3}\left(t_{f}\right)+\lambda_{9}\left(t_{f}\right)=0
\end{aligned}
$$

Then applying the function extremum theory to system Hamiltonian function $H_{P}$, the co-state equations are derived as the following

$$
\begin{aligned}
& \dot{\boldsymbol{\lambda}}_{P}=-\left[H_{\boldsymbol{x}_{P}}\right]^{T}=-\left[\frac{\partial H_{P}}{\partial \boldsymbol{x}_{P}}\right]^{T}=-\left[\frac{\partial \boldsymbol{f}_{P}}{\partial \boldsymbol{x}_{P}}\right]^{T} \lambda_{P} \\
& \dot{\lambda}_{E}=-\left[H_{\boldsymbol{x}_{E}}\right]^{T}=-\left[\frac{\partial H_{E}}{\partial \boldsymbol{x}_{E}}\right]^{T}=-\left[\frac{\partial \boldsymbol{f}_{E}}{\partial \boldsymbol{x}_{E}}\right]^{T} \lambda_{E}
\end{aligned}
$$

And using $\mathrm{s}(\cdot)$ and $\mathrm{c}(\cdot)$ with replacing $\sin (\cdot)$ and $\cos (\cdot)$ respectively and substituting $\boldsymbol{f}_{P}$ as equations (6), then the co-state equations above can be written as equation (17). 


$$
\begin{aligned}
& \dot{\lambda}_{1}=-\lambda_{2} \frac{x_{5} \mathrm{c} x_{4} \mathrm{c} x_{6}}{x_{1}^{2} \mathrm{c} x_{3}}-\lambda_{3} \frac{x_{5} \mathrm{c} x_{4} \mathrm{~s} x_{6}}{x_{1}^{2}}+\lambda_{4}\left(\frac{3 G_{J 2}}{x_{1}^{5} x_{5}}\left\{\mathrm{~s} x_{4}\left[3 \mathrm{c}\left(2 x_{3}\right)-2\right]+2 \mathrm{~s}\left(2 x_{3}\right) \mathrm{c} x_{5} \mathrm{~s} x_{6}\right\}\right. \\
& \left.\frac{2 \mu \mathrm{c} x_{4}}{x_{1}^{3} x_{5}}-\frac{x_{5} \mathrm{c} x_{4}}{x_{1}^{2}}\right)+\lambda_{5}\left(\frac{3 G_{J 2}}{x_{1}^{5}}\left\{\mathrm{~s} x_{4}\left[3 \mathrm{c}\left(2 x_{3}\right)-2\right]+2 \mathrm{~s}\left(2 x_{3}\right) \mathrm{c} x_{5} \mathrm{~s} x_{6}\right\}+\frac{2 \mu \mathrm{s} x_{4}}{x_{1}^{3}}\right) \\
& +\lambda_{6}\left[\frac{x_{5} \mathrm{c} x_{4} \mathrm{c} x_{6} \mathrm{~s} x_{3}}{x_{1}^{2} \mathrm{c} x_{3}}+\frac{6 G_{J 2} \mathrm{~s}\left(2 x_{3}\right) \mathrm{c} x_{6}}{x_{1}^{5} x_{5} \mathrm{c} x_{4}}\right] \\
& \dot{\lambda}_{2}=0 \\
& \dot{\lambda}_{3}=\lambda_{2} \frac{x_{5} \mathrm{c} x_{4} \mathrm{~s} x_{3}}{x_{1} \mathrm{c}^{2} x_{3}}+\lambda_{4} \frac{3 G_{J 2}}{4 x_{1}^{4} x_{5}}\left[6 \mathrm{~s}\left(2 x_{3}\right) \mathrm{c} x_{4}+4 \mathrm{c}\left(2 x_{3}\right) \mathrm{s} x_{4} \mathrm{~s} x_{6}\right]+\lambda_{5} \frac{3 G_{J 2}}{4 x_{1}^{4}}\left[6 \mathrm{~s}\left(2 x_{3}\right) \mathrm{s} x_{4}\right. \\
& \left.-4 \mathrm{c}\left(2 x_{3}\right) \mathrm{c} x_{5} \mathrm{~s} x_{6}\right]-\lambda_{6}\left[\frac{x_{5} \mathrm{c} x_{4} \mathrm{c} x_{6}}{x_{1}}\left(1+\frac{\mathrm{s}^{2} x_{3}}{\mathrm{c}^{2} x_{3}}\right)+\frac{3 G_{J 2} \mathrm{c}\left(2 x_{3}\right) \mathrm{c} x_{6}}{x_{1}^{4} x_{5} \mathrm{c} x_{4}}\right] \\
& \dot{\lambda}_{4}=\lambda_{1} x_{5} \mathrm{c} x_{4}-\lambda_{2} \frac{x_{5} \mathrm{c} x_{6} \mathrm{~s} x_{4}}{x_{1} \mathrm{c} x_{3}}-\lambda_{3} \frac{x_{5}}{x_{1}} \mathrm{~s} x_{4} \mathrm{~s} x_{6}+\lambda_{4}\left(\frac{\mu \mathrm{s} x_{4}}{x_{1}^{2} x_{5}}+\frac{3 G_{J 2}}{4 x_{1}^{4} x_{5}}\left\{\mathrm{~s} x_{4}\left[3 \mathrm{c}\left(2 x_{3}\right)-1\right]\right.\right. \\
& \left.\left.+2 \mathrm{~s}\left(2 x_{3}\right) \mathrm{c} x_{4} \mathrm{~s} x_{6}\right)-\frac{x_{5} \mathrm{~s} x_{4}}{x_{1}}\right)-\lambda_{5}\left(\frac{3 G_{J 2}}{4 x_{1}^{4}} \mathrm{c} x_{4}\left[3 \mathrm{c}\left(2 x_{3}\right)-1\right]+\frac{\mu \mathrm{c} x_{4}}{x_{1}^{2}}\right) \\
& +\lambda_{6}\left[\frac{U_{E} \mathrm{~s} \beta_{P} \mathrm{~s} x_{4}}{x_{5} \mathrm{c}^{2} x_{4}}+\frac{x_{5} \mathrm{c} x_{6} \mathrm{~s} x_{3} \mathrm{~s} x_{4}}{x_{1} \mathrm{c} x_{3}}-\frac{3 G_{J 2} \mathrm{~s}\left(2 x_{3}\right) \mathrm{c} x_{6} \mathrm{~s} x_{4}}{2 x_{1}^{4} x_{5} \mathrm{c}^{2} x_{4}}\right] \\
& \dot{\lambda}_{5}=\lambda_{1} \mathrm{~s} x_{4}+\lambda_{2} \frac{\mathrm{c} x_{4} \mathrm{c} x_{6}}{x_{1} \mathrm{c} x_{3}}+\lambda_{3} \frac{\mathrm{c} x_{4} \mathrm{~s} x_{6}}{x_{1}}+\lambda_{4}\left(\frac{\mathrm{c} x_{4}}{x_{1}}-\frac{U_{P} \mathrm{~s} \alpha_{P} \mathrm{c} \beta_{P}}{x_{5}^{2}}+\frac{3 G_{J 2}}{4 x_{1}^{4} x_{5}^{2}}\left\{-2 \mathrm{~s}\left(2 x_{3}\right) \mathrm{c} x_{4} \mathrm{~s} x_{6}\right\}+\frac{\mu \mathrm{c} x_{4}}{x_{1}^{4} x_{5}^{2}}\right) \\
& -\lambda_{5} \frac{3 G_{J 2}}{2 x_{1}^{4}} \mathrm{~s}\left(2 x_{3}\right) \mathrm{s} x_{4} \mathrm{~s} x_{6}-\lambda_{6}\left[\frac{U_{P} \mathrm{~s} \beta_{P}}{x_{5}^{2} \mathrm{c} x_{4}}+\frac{\mathrm{c} x_{4} \mathrm{c} x_{6} \mathrm{~s} x_{3}}{x_{1} \mathrm{c} x_{3}}-\frac{3 G_{J 2} \mathrm{~s}\left(2 x_{3}\right) \mathrm{c} x_{6} \mathrm{~s} x_{4}}{2 x_{1}^{4} x_{5}^{2} \mathrm{c} x_{4}}\right] \\
& \dot{\lambda}_{6}=-\lambda_{2} \frac{x_{5} \mathrm{c} x_{4} \mathrm{~s} x_{6}}{x_{1} \mathrm{c} x_{3}}+\lambda_{3} \frac{x_{5} \mathrm{c} x_{4} \mathrm{c} x_{6}}{x_{1}}+\lambda_{4} \frac{3 G_{J 2} \mathrm{~s}\left(2 x_{3}\right) \mathrm{s} x_{4} \mathrm{c} x_{6}}{2 x_{1}^{4} x_{5}} \\
& -\lambda_{5} \frac{3 G_{J 2} \mathrm{~s}\left(2 x_{3}\right) \mathrm{c} x_{4} \mathrm{c} x_{6}}{2 x_{1}^{4}}+\lambda_{6}\left[\frac{x_{5} \mathrm{c} x_{4} \mathrm{~s} x_{6} \mathrm{~s} x_{3}}{x_{1} \mathrm{c} x_{3}}+\frac{3 G_{J 2} \mathrm{~s}\left(2 x_{3}\right) \mathrm{s} x_{6}}{2 x_{1}^{4} x_{5} \mathrm{c} x_{4}}\right]
\end{aligned}
$$

The terminal time of interception is uncertain, thus the system Hamilton satisfies the following terminal condition

$$
\left.H\right|_{t=t_{f}}+\left[\frac{\partial \varphi\left(t_{f}, \boldsymbol{x}\left(t_{f}\right)\right)}{\partial t_{f}}+\boldsymbol{v}^{T} \frac{\partial \boldsymbol{N}}{\partial t}\right]=\left.H\right|_{t=t_{f}}+1=0
$$

The Hamilton function can be divided to two parts $H_{P}$ and $H_{E}$, so applying the min-max condition to $H$ is equal to deriving the minimum of $H_{P}$ and maximum of $H_{E}$. Then the optimal control strategies of the pursuer and evader satisfy the equations as below

$$
\begin{aligned}
\boldsymbol{u}_{P}^{*}=\arg \min _{\boldsymbol{u}_{P}} H=\arg \min _{\boldsymbol{u}_{P}} H_{P} \\
=\arg \min _{\left[\alpha_{P}, \beta_{P}\right]}\left[\begin{array}{l}
\lambda_{4} \frac{\sin \alpha_{P} \cos \beta_{P}}{v_{P}}+\lambda_{6} \frac{\sin \beta_{P}}{v_{P} \cos \gamma_{P}} \\
+\lambda_{5} \cos \alpha_{P} \cos \beta_{P}+(\cdot)_{P}
\end{array}\right] \\
\boldsymbol{u}_{E}^{*}=\arg \max _{\boldsymbol{u}_{E}} H=\arg \max _{\boldsymbol{u}_{E}} H_{E} \\
=\arg \max _{\left[\alpha_{E}, \beta_{E}\right]}\left[\begin{array}{l}
\lambda_{10} \frac{\sin \alpha_{E} \cos \beta_{E}}{v_{E}}+\lambda_{12} \frac{\sin \beta_{E}}{v_{E} \cos \gamma_{E}} \\
+\lambda_{11} \cos \alpha_{E} \cos \beta_{E}+(\cdot)_{E}
\end{array}\right]
\end{aligned}
$$


where $(\cdot)_{P}$ and $(\cdot)_{E}$ represent the remainders which is irrelevant to the control angles of the pursuer and evader, respectively. Continuing the derivation process, the optimal controls of both sides $\left(\left[\alpha_{P}^{*}, \beta_{P}^{*}\right],\left[\alpha_{E}^{*}, \beta_{E}^{*}\right]\right)$ can be obtained as equation $(22)$.

$$
\left\{\begin{array}{l}
\beta_{P}^{*}=-\arcsin \frac{\lambda_{6}}{v_{P} \cos \gamma_{P}}\left[\left(\frac{\lambda_{4}}{v_{P}}\right)^{2}+\lambda_{5}^{2}+\left(\frac{\lambda_{6}}{v_{P} \cos \gamma_{P}}\right)^{2}\right]^{-\frac{1}{2}} \\
\cos \alpha_{P}^{*}=-\frac{\lambda_{5}}{\cos \beta_{P}^{*}}\left[\left(\frac{\lambda_{4}}{v_{P}}\right)^{2}+\lambda_{5}^{2}+\left(\frac{\lambda_{6}}{v_{P} \cos \gamma_{P}}\right)^{2}\right]^{-\frac{1}{2}} \\
\sin \alpha_{P}^{*}=-\frac{\lambda_{4}}{v_{P} \cos \beta_{P}^{*}}\left[\left(\frac{\lambda_{4}}{v_{P}}\right)^{2}+\lambda_{5}^{2}+\left(\frac{\lambda_{6}}{v_{P} \cos \gamma_{P}}\right)^{2}\right]^{-\frac{1}{2}}
\end{array},\left\{\begin{array}{l}
\cos \alpha_{E}^{*}=\frac{\lambda_{11}}{\cos \beta_{E}^{*}}\left[\left(\frac{\lambda_{10}}{v_{E}}\right)^{2}+\lambda_{11}^{2}+\left(\frac{\lambda_{12}}{v_{E} \cos \gamma_{E}}\right)^{2}\right]^{2}+\lambda_{11}^{2}+\left(\frac{1}{v_{E} \cos \gamma_{E}}\right)^{-\frac{1}{2}} \\
\sin \alpha_{E}^{*}=\frac{\lambda_{10}}{v_{E} \cos \beta_{E}^{*}}\left[\left(\frac{\lambda_{10}}{v_{E}}\right)^{2}+\lambda_{11}^{2}+\left(\frac{\lambda_{12}}{v_{E} \cos \gamma_{E}}\right)^{2}\right]^{-\frac{1}{2}}
\end{array}\right.\right.
$$

\section{Satellites Pursuit and Evasion solving based on Mixed Optimization}

Obviously if the conjugates at any time are known, the control angles can be derived from equation (22). However the former needs to be integrated from the starting of interception with known initials. In order to solve this problem, it was performed to collect the system (6) and conjugate (17) to a set of differential equations and combine them with equations (15), (17) and (18) to form a two point boundary value problem. Thus the problem has 13 equality constraints and 13 knowns with including 12 initial conjugates and the interception ending time. Normally, the TPBVP is difficult to solve analytically for the nonlinearity of system dynamics. Numerical methods like shooting and difference are usually used to derive the solution. And good initial guess is required to guarantee the convergence and correctness of the solving process. In order to avoid these faults, the TPBVP was transformed to an unconstrained optimization problem where the design variables are the 13 knowns and the objective was constructed by minimize the distance between the function values formed from the equalities constraints (15), (17) and (18) and the original. The distance driving to zero in the optimize process equals to the satisfaction of the equalities constraints.

A mixed optimization method consisting of two stages was used in solving the optimization problem described above. Firstly, we make use of heuristic optimization method to search the approximated solution in the globally optimum's convergent region. In the second step, the sequential quadratic programming was employed to accurately refine the approximated solution. Based on the second order Taylor approximation, the SQP algorithm calculates the gradients and Hessian matrix of the objective function sequentially, and transform the preview complex constrained optimization problem to a series linear or quadratic regulating problems which satisfy the convex optimization theory. Solution of the original question was corrected after this sub-optimization problem solved.

Heuristic optimization imitates the natural evolving process, including genetic algorithm, simulated annealing algorithm, swarm algorithm, cuckoo search, etc. This paper employed the genetic algorithm as reference [15]. So the objective construction was same. But in the second step, the semidirect method was changed by directly optimize the constructed objective in the first stage with the safe altitude constraints on the attacking-escaping satellites like the below

$$
\begin{aligned}
& \left(r_{e}+h_{\text {safe }}\right)-r_{P} \leq 0 \\
& \left(r_{e}+h_{\text {safe }}\right)-r_{E} \leq 0
\end{aligned}
$$

where $h_{\text {safe }}$ is the safe altitude. The inequalities above require the radial distance of both player should not be less than a predefine value to keep them away from intersecting the atmosphere layer. 


\section{Simulations and Two Side Optimal Trajectories}

The optimization method described in section 4 was applied to solve the pursuit-evasion game of satellites with J2 non-spherical perturbation. Dimensional normalization was implemented as the reference [18] where the normalized length and time were given as $\mathrm{DU}=1 R_{E}$ and $\mathrm{TU}=806.8 \mathrm{~s}$ respectively. Then the normalized acceleration derived from the DU and TU would be $1 \mathrm{AU}=9.798 \cdot 10^{-3} \mathrm{~km} / \mathrm{s}^{2}$. $h_{\text {safe }}$ equals to $100 \mathrm{~km}$. Satellites orbital elements of the pursuer and evader were given in table 1 .

Table 1. Orbital elements of the purser and evader

\begin{tabular}{cccccc}
\hline $\begin{array}{c}\text { Orbital } \\
\text { elements }\end{array}$ & $a /(\mathrm{km})$ & $e$ & $i /\left(^{\circ}\right)$ & $\Omega /\left(^{\circ}\right)$ & $M /\left(^{\circ}\right)$ \\
\hline pursuer & $\mathbf{6 9 7 8 . 1 6 5}$ & $\mathbf{0}$ & $\mathbf{4 0}$ & $\mathbf{0}$ & $\mathbf{3 1 0}$ \\
evader & $\mathbf{6 9 7 8 . 1 6 5}$ & $\mathbf{0}$ & $\mathbf{6 0}$ & $\mathbf{3 0}$ & $\mathbf{2 9 0}$ \\
\hline
\end{tabular}

The intercepting and target satellites moved in the low orbits with different inclinations. The accelerations of them were given as $U_{P}=0.02 \mathrm{AU}, U_{E}=0.01 \mathrm{AU}$. The genetic algorithm was embodied by the $g a$ function in Matlab. The inherit-selection process continues 2000 generations and each generation has 100 individuals. Also cross and mutation probability were set to be 0.8 and 0.2 respectively. The SQP accurate solution was achieved by the Matlab function fmincon. The genetic search process goes 17 minutes in total. Lastly the optimization gave the solution with normalized interception ending time being 2.482 . The final optimized costate variables were given in table 2 .

Table 2. Costate variables

\begin{tabular}{cccccccccccc}
\hline$\lambda_{1}$ & $\lambda_{2}$ & $\lambda_{3}$ & $\lambda_{4}$ & $\lambda_{5}$ & $\lambda_{6}$ & $\lambda_{7}$ & $\lambda_{8}$ & $\lambda_{9}$ & $\lambda_{10}$ & $\lambda_{11}$ & $\lambda_{12}$ \\
\hline $\mathbf{2 . 9 4 8}$ & $\mathbf{- 0 . 2 0 6}$ & $\mathbf{- 0 . 3 1 6}$ & $\mathbf{- 2 . 3 6 6}$ & $\mathbf{3 . 4 2 0}$ & $\mathbf{- 2 . 1 5 2}$ & $\mathbf{- 4 . 1 1 6}$ & $\mathbf{0 . 0 6 1}$ & $\mathbf{0 . 1 4 7}$ & $\mathbf{0 . 5 6 2}$ & $\mathbf{3 . 6 0 3}$ & $\mathbf{- 4 . 9 9 7}$ \\
\hline
\end{tabular}

Substituting the initials of co-states and system states to the integration of the conjugate and dynamic equation, the states variations of the pursuer and evader were obtained, as shown in figure 1 .

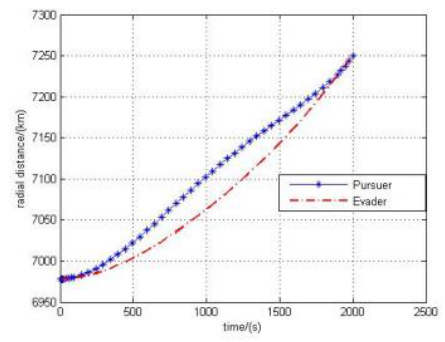

a. Radial distance

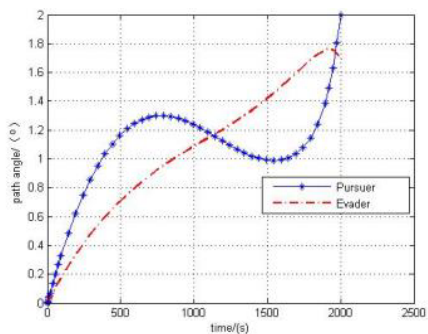

d. Path angle

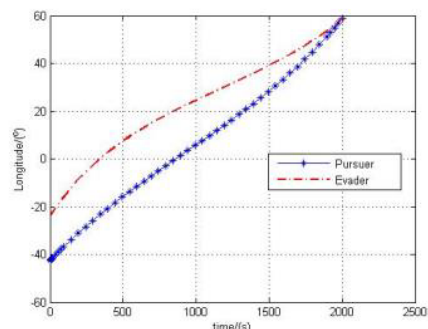

b. geometrical longitude

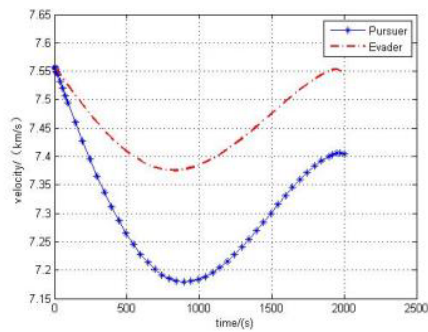

e. Velocity

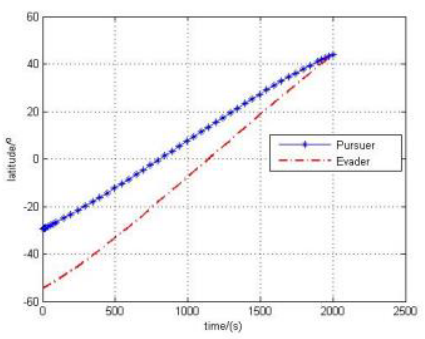

c. Geometrical latitude

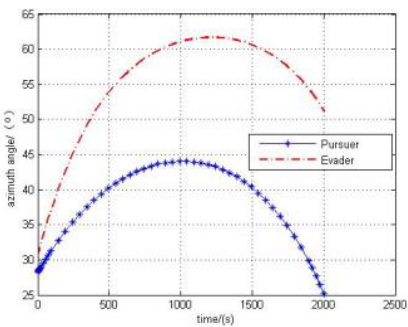

f. Azimuth angle 
Figure 1. States of pursuer and evader

From the figures, it was found that the pursuer successfully intercept the evader when the orbital attitude get $872 \mathrm{~km}$, geometrical longitude and latitude arrive at $60^{\circ}$ and $42^{\circ}$ after 2002 seconds. The optimal trajectories of the interceptor and escaper are given in figure 2.

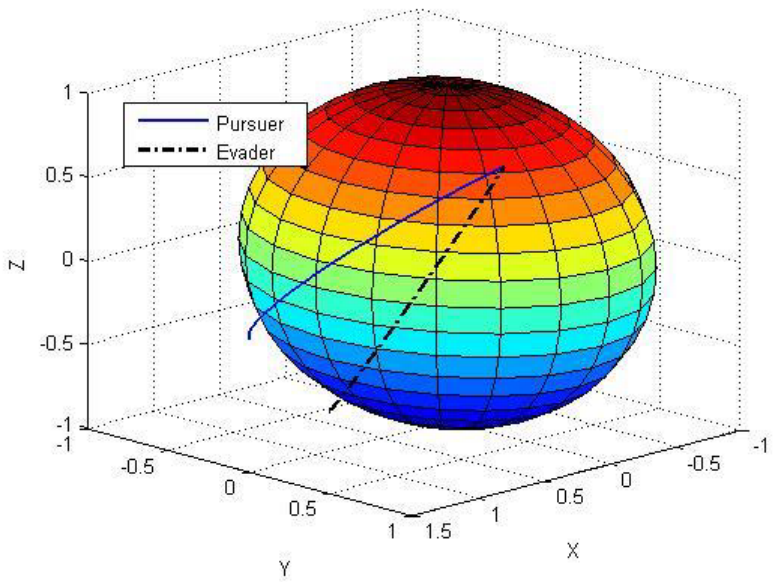

Figure 2. Trajectories of the pursuer and evader

\section{Conclusion}

In the present paper the typical satellite interception problem was revised, where both the pursuer and the evader can apply the continuous thrust to perform orbital maneuver in the earth central force with the $\mathrm{J} 2$ non-spherical perturbation. The two-side optimization was involved since the interceptor and the escaper will try their best to compete in the attacking-defense scenario. The satellite dynamic with J2 perturbation was derived. The function extremum theory was used to obtain the necessary condition of the Hamilton system and the optimal control strategies of both sides when the pursuer and evader game have got its saddle solution. Then the pursuit-evasion game was turned into a two point boundary value problem and a further constrained optimization problem. Genetic algorithm was employed to search the approximation of the global optimal solution. The accurate solution was achieved by application of the SQP method to re-optimize the objective with the approximating solution as starting initials. Lastly, the two side optimal control angles and the optimal trajectories of the pursuer and evader were simulated and calculated to validate the efficiency of the method.

\section{References}

1. Luo, Y. Z., Tang, G. J., \& Li, H. Y. Optimization of multiple-impulse minimum-time rendezvous with impulse constraints using a hybrid genetic algorithm [J]. Aerospace Science and Technology, 2006, 10(6): 534-540.

2. Hughes, S. P., Mailhe, L. M., \& Guzman, J. J. A comparison of trajectory optimization methods for the impulsive minimum fuel rendezvous problem[C]. In 26th Rocky Mountain Guidance and Control Conference. 2003: 3-6.

3. Luo, Y. Z., Li, H. Y., \& Tang, G. J. Hybrid approach to optimize a rendezvous phasing strategy[J]. Journal of Guidance, Control and Dynamics, 2007, 30(1): 185-191.

4. Luo, Y. Z., \& Tang, G. J. Spacecraft optimal rendezvous controller design using simulated annealing[J]. Aerospace Science and Technology, 2005, 9(8): 732-737. 
5. Luo, Y. Z., Tang, G. J., Li, Y. J., \& Li, H. Y. Optimization of multiple-impulse, multiplerevolution, rendezvous-phasing maneuvers[J]. Journal of Guidance, Control, and Dynamics, 2007, 30(4): 946-952.

6. F. Lawden. Optimal Trajectories for Space Navigation[J]. London Butter Worths. 1963

7. P. M. Lion, M. Handelsman.Primer Vector on Fixed-Time Impulsive Trajectories [J].AIAA Journal. 1968, 6(1): 127-132.

8. Greenwood D T, Kern E A. Minimum-fuel thrust limited transfer trajectories between coplanar elliptic orbits[J]. AIAA Journal, 1970, 8(10): 1772-1779.

9. Hargraves C R, Paris S W. Direct trajectory optimization using nonlinear pro-gramming and collocation[J]. Journal of Guidance, Control, and Dynamics, 1987, 10(4): 338-342.

10. Enright P J, Conway B A. Discrete approximations to optimal trajectories using direct transcription and nonlinear programming[J]. Journal of Guidance, Control, and Dynamics, 1992, 15(4): 994-1002.

11. Herman A L, Spencer D B. Optimal, low-thrust earth-orbit transfers using higher- order collocation methods[J]. Journal of Guidance, Control, and Dynamics, 2002, 25(1): 40-47.

12. Isaacs R. Differential games: a mathematical theory with applications to warfare and pursuit, control and optimization[M]. Courier Dover Publications, 1999. 3-43

13. Anderson G M. Feedback control for a pursuing spacecraft using differential dynamic programming[J]. AIAA Journal, 1977, 15(8): 1084-1088.

14. Menon P K A, Calise A J, Leung S K M. Guidance laws for spacecraft pursuit-evasion and rendezvous[C]//AIAA Guidance Navigation and Control. 1988, 668-697.

15. Mauro Pontani, Bruce A. Conway. Optimal Interception of Evasive Missile Warheads: Numerical Solution of the Differential Game[J]. Journal of Guidance, Control, and Dynamics, 2008, 31(4): 1111-1122.

16. Mauro Pontani, Bruce A. Conway. Numerical Solution of the Three-Dimensional Orbital PursuitEvasion Game Orbital Pursuit-Evasion Game[J]. Journal of Guidance, Control, and Dynamics, 2009, 32(2): 474-487.

17. Stupik J, Pontani M, Conway B. Optimal pursuit-evasion spacecraft trajectories in the hill reference frame[C]//AIAA/AAS Astrodynamics Specialist Conference. Reston, VA: AIAA, 2012: 4882.

18. Pontani M. Numerical Solution of Orbital Combat Games Involving Missiles and Spacecraft[J]. Dynamic Games and Applications, 2011, 1(4): 534-557. 